\title{
Inner Automorphisms of von Neumann Algebras
}

\author{
Erling Størmer \\ University of Oslo, Oslo, Norway \\ Received October 15, 1973
}

\begin{abstract}
It is first shown that a *automorphism of a factor is inner if and only if it is asymptotically equal to the identity automorphism. Then it is shown that a periodic *-automorphism of a von Neumann algebra $\mathscr{R}$ is inner if and only if its fixed point algebra is a normal subalgebra of $\mathscr{R}$.
\end{abstract}

\section{Introduction}

It is often of importance to know whether a *automorphism of a von Neumann algebra is inner or not. In the present paper we shall study two aspects of this problem. The first results essentially state that a *-automorphism $\alpha$ of a factor $\mathscr{R}$ is inner if and only if it is asymptotically equal to the identity automorphism $l$. By this we mean that if $\varepsilon>0$ is sufficiently small, then there is a type $I$ subfactor $m$ of $\mathscr{R}$ such that $\left\|(\alpha-\imath) \mid m^{c}\right\|<\varepsilon$, where $m^{c}=m^{\prime} \cap \mathscr{R}$. A similar theorem has been obtained by Lance [7] for UHF-algebras. The second set of results combine innerness with properties of the fixed point algebra $\mathscr{R}^{\alpha}$ of $\alpha$. The main result says that a necessary and sufficient condition for a periodic $\alpha$ to be inner is that $\mathscr{R}^{\alpha}$ is normal in $\mathscr{R}$, i.e. $\mathscr{R}^{\alpha}=\mathscr{R}^{\alpha c c}$. The first results are for simplicity stated for factors while the latter are proved for general von Neumann algebras.

\section{Asymptotic Properties}

In this section we prove the asymptotic theorems mentioned in the introduction. The key result is the following lemma; $l$ will here and later denote the identity automorphism.

Lemma 2.1. Let $\mathscr{R}$ be a factor, $\alpha$ a *automorphism of $\mathscr{R}$, and $0<\varepsilon<1 / 1800$. Suppose there is a type I subfactor $m$ of $\mathscr{R}$ such that $\left\|(\alpha-\imath) \mid m^{c}\right\|<\varepsilon$. Then $\alpha$ is inner.

Proof. We first show $\|m-\alpha(m)\| \leqq 6 \varepsilon$, where $\|\mathfrak{A}-\mathscr{B}\|$ denotes the distance between two *-algebras, i.e.

$$
\|\mathfrak{U}-\mathscr{B}\|=\sup \left\{\delta\left(A, \mathscr{B}_{1}\right), \delta\left(B, \mathfrak{A}_{1}\right):\|A\| \leqq 1, A \in \mathfrak{A},\|B\| \leqq 1, B \in \mathscr{B}\right\}
$$

where $\delta\left(A, \mathscr{B}_{1}\right)=\inf \{\|A-B\|: B \in \mathscr{B},\|B\| \leqq 1\}$, see [5]. 
Let $A \in \alpha(m),\|A\| \leqq 1$. Let $B \in m^{c}$. Then, since $\alpha^{-1}(A) \in m$, we have

$$
\begin{aligned}
\|[A, B]\|= & \left\|\left[\alpha^{-1}(A), \alpha^{-1}(B)\right]\right\| \\
\leqq & \|\left[\alpha^{-1}(A) \alpha^{-1}(B)-\alpha^{-1}(A) B\|+\| \alpha^{-1}(A) B-B \alpha^{-1}(A) \|\right. \\
& +\left\|B \alpha^{-1}(A)-\alpha^{-1}(B) \alpha^{-1}(A)\right\| \\
\leqq & 2\left\|\alpha^{-1}(A)\right\|\left\|B-\alpha^{-1}(B)\right\| \\
\leqq & 2 \varepsilon\|B\| .
\end{aligned}
$$

In particular, if $U$ is a unitary operator in $m^{c}$, then

$$
\left\|U A U^{-1}-A\right\|=\|U A-A U\| \leqq 2 \varepsilon .
$$

More generally, if $U_{k}$ are unitary operators in $m^{c}, \lambda_{k}>0, \Sigma \lambda_{k}=1$, for $k=1,2, \ldots, n$, then we have

$$
\left\|\Sigma \lambda_{k} U_{k} A U_{k}^{-1}-A\right\| \leqq \Sigma \lambda_{k}\left\|U_{k} A U_{k}^{-1}-A\right\| \leqq 2 \varepsilon .
$$

Suppose we have shown $\delta\left(P A P, m_{1}\right)<6 \varepsilon$ for each finite dimensional projection $P \in m$. Then $\delta\left(A, m_{1}\right) \leqq 6 \varepsilon$. Indeed, let $\left\{P_{\gamma}\right\}$ be an increasing net of finite dimensional projections in $m$ converging strongly to the identity $I$. For each $\gamma$ we can choose $B_{\gamma} \in m_{1}$ such that $\left\|P_{\gamma} A P_{\gamma}-B_{\gamma}\right\|<6 \varepsilon$. Since the unit ball is weakly compact in $m$ we can choose a subnet $\left\{B_{\beta}\right\}$ of $\left\{B_{\gamma}\right\}$ converging weakly to an operator $B \in m_{1}$. Since $\left\{P_{\beta}\right\}$ is a subnet of the converging net $\left\{P_{\gamma}\right\}, P_{\beta} \rightarrow I$ strongly, hence $P_{\beta} A P_{\beta} \rightarrow A$ strongly. Let $x$ and $y$ be vectors in the underlying Hilbert space. Then

$$
\begin{aligned}
|((B-A) x, y)| & =\lim _{\beta}\left|\left(\left(B_{\beta}-P_{\beta} A P_{\beta}\right) x, y\right)\right| \\
& \leqq \varlimsup_{\beta}\left\|B_{\beta}-P_{\beta} A P_{\beta}\right\|\|x\|\|y\| \\
& \leqq 6 \varepsilon\|x\|\|y\| .
\end{aligned}
$$

Thus $\|B-A\| \leqq 6 \varepsilon$, and $\delta\left(A, m_{1}\right) \leqq 6 \varepsilon$ as asserted.

Let $P$ be a finite dimensional projection in $m$, and let $\left\{e_{i j}\right\}_{i, j=1, \ldots, n}$ be a complete set of matrix units in $P m P$. Since $\mathscr{R}$ is generated by $m$ and $m^{c}$ we may write $P A P=\Sigma e_{i j} A_{i j}$, where $A_{i j} \in m^{c}$. By [4, Chapter III, $\S 5$, Lemma 5] there exist complex numbers $a_{i j}$, unitary operators $U_{k}$ in $m^{c}$, and $\lambda_{k}>0$ satisfying $\Sigma \lambda_{k}=1, k=1, \ldots, m$, such that

$$
\left\|\sum_{k} \lambda_{k} U_{k} A_{i j} U_{k}^{-1}-a_{i j} I\right\|<\varepsilon / n^{2} .
$$


Thus by (1) we have

$$
\begin{aligned}
& \left\|P A P-\sum_{i j} a_{i j} e_{i j}\right\| \leqq\left\|P A P-\sum_{k} \lambda_{k} U_{k} P A P U_{k}^{-1}\right\| \\
& +\left\|\sum_{i j k} \lambda_{k} e_{i j} U_{k} A_{i j} U_{k}^{-1}-\sum_{i j} a_{i j} e_{i j}\right\| \\
\leqq & \left\|P\left(A-\sum_{k} \lambda_{k} U_{k} A U_{k}^{-1}\right) P\right\|+\sum_{i j}\left\|\sum_{k} \lambda_{k} U_{k} A_{i j} U_{k}^{-1}-a_{i j} I\right\| \\
< & 2 \varepsilon+n^{2}\left(\varepsilon / n^{2}\right)=3 \varepsilon .
\end{aligned}
$$

In particular $\left\|\sum_{i j} a_{i j} e_{i j}\right\|<1+3 \varepsilon$, and $\left\|P A P-(1+3 \varepsilon)^{-1} \sum_{i j} a_{i j} e_{i j}\right\|<6 \varepsilon$. Since $\sum a_{i j} e_{i j} \in m$, we have shown $\delta\left(P A P, m_{1}\right)<6 \varepsilon$, so $\delta\left(A, m_{1}\right) \leqq 6 \varepsilon$ by the above paragraph. By a symmetric argument $\delta\left(B, \alpha\left(m_{1}\right)\right) \leqq 6 \varepsilon$ for $B \in m,\|B\| \leqq 1$. Thus $\|m-\alpha(m)\| \leqq 6 \varepsilon$, as asserted.

By a result of Christensen [2] there is a unitary operator $U \in\{m \cup \alpha(m)\}^{\prime \prime} \subset \mathscr{R}$ such that $\|I-U\|<299 \cdot(6 \varepsilon)=1794 \varepsilon$, and such that $U m U^{-1}=\alpha(m)$. Let $\beta(A)=U^{-1} \alpha(A) U$ for $A \in \mathscr{R}$. Then $\beta$ is a *-automorphism of $\mathscr{R}$ such that $\beta(m)=m$, hence $\beta\left(m^{c}\right)=m^{c}$. If $A \in m^{c}$ is nonzero, we have

$$
\begin{aligned}
\|(\beta-\imath)(A)\| & \leqq\left\|U^{-1} \alpha(A) U-\alpha(A)\right\|+\|\alpha(A)-A\| \\
& <2\|U-I\|\|\alpha(A)\|+\varepsilon\|A\| \\
& <(2 \cdot 1794 \varepsilon+\varepsilon)\|A\| \\
& <2\|A\| .
\end{aligned}
$$

Thus $\left\|(\beta-\imath) \mid m^{c}\right\|<2$, so by a result of Kadison and Ringrose [6] $\beta \mid m^{c}$ is inner. Let $W$ be a unitary operator in $m^{c}$ such that $\beta(A)=W A W^{-1}$ for $A \in m^{c}$. Since $\beta \mid m$ is a *automorphism, and $m$ is a type $I$ factor, there is a unitary operator $V$ in $m$ such that $\beta(A)=V A V^{-1}$ for $A \in m$ [4, Chapter III, $\S 3$, Proposition 4]. Now $\mathscr{R}$ equals the von Neumann algebra generated by $m$ and $m^{c}$, and if $A \in m, B \in m^{c}$ we have $\beta(A B)=\beta(A) \beta(B)=V A V^{-1} W B W^{-1}=V W A B W^{-1} V^{-1}, \quad$ so $\quad \beta(C)$ $=V W C W^{-1} V^{-1}$ for all $C \in \mathscr{R}$. Let $X=U V W$. Then $X$ is a unitary operator in $\mathscr{R}$, and if $A \in \mathscr{R}$ then

$$
X A X^{-1}=U V W A W^{-1} V^{-1} U^{-1}=U \beta(A) U^{-1}=\alpha(A) .
$$

Thus $\alpha$ is implemented by $X$, and $\alpha$ is inner. The proof is complete.

The converse of the above lemma is practically true. The only difficulty occurs in the case when $\mathscr{R}$ is of type II. Suppose for example $\alpha$ is implemented by a unitary operator $U$ in $\mathscr{R}$ of the form $U=E-F$, where $E$ and $F$ are finite projections in $\mathscr{R}$ such that $\operatorname{dim} E / \operatorname{dim} F$ is irrational. Then it is not clear that we can obtain the conditions in the 
lemma. We shall therefore need to approximate those conditions. First, however, we treat the in this case simple type III situation.

Theorem 2.2. Let $\mathscr{R}$ be a factor of type III, $\alpha$ a*-automorphism of $\mathscr{R}$, and $0<\varepsilon<1 / 1800$. Then $\alpha$ is inner if and only if there is a type I subfactor $m$ of $\mathscr{R}$ such that $\left\|(\alpha-\imath) \mid m^{c}\right\|<\varepsilon$.

Proof. By Lemma 2.1 it remains to show the necessity. Let $\alpha(A)$ $=U A U^{-1}$ for all $A \in \mathscr{R}$ with $U$ a unitary operator in $\mathscr{R}$. By spectral theory there are spectral projections $E_{1}, \ldots, E_{n}$ for $U$ in $\mathscr{R}$ with sum I such that $\left\|U-\sum_{k} \lambda_{k} E_{k}\right\|<\varepsilon / 2$ for some complex numbers $\lambda_{1}, \ldots, \lambda_{n}$ of modulus 1 . Each projection $E$ in $\mathscr{R}$ is an orthogonal sum of countably decomposable projections in $\mathscr{R}$. Indeed, we can find a family $\left\{\omega_{\gamma}\right\}$ of vector states of $\mathscr{R}$ with mutually orthogonal supports with sum $E$. But the support of a vector state is countably decomposable. We can therefore decompose the projections $E_{1}, \ldots, E_{n}$ into orthogonal sums of countably decomposable projections $\left\{F_{\gamma}\right\}$. Since all nonzero countably decomposable projections in $\mathscr{R}$ are equivalent $[4$, Chapter III, $\S 8$, Corollary 5], we can find a type I subfactor $m$ of $\mathscr{R}$ containing all the $F_{\gamma}$. Since the automorphism implemented by $\Sigma \lambda_{k} E_{k}$ is the identity on $\mathrm{m}^{c}$, $\left\|(\alpha-l) \mid m^{c}\right\| \leqq 2\left\|U-\sum_{k} \lambda_{k} E_{k}\right\|<2 \cdot \varepsilon / 2=\varepsilon$. The proof is complete.

From the above proof we have.

Corollary 2.3. Let $\mathscr{R}$ be a countably decomposable factor of type III, $\alpha a^{*}$-automorphism of $\mathscr{R}$ and $0<\varepsilon<1 / 1800$. Then $\alpha$ is inner if and only if there is a finite type I subfactor $m$ of $\mathscr{R}$ such that $\left\|(\alpha-\imath) \mid m^{c}\right\|<\varepsilon$.

If $\alpha$ is a *automorphism of a von Neumann algebra $\mathscr{R}$ we denote by $\mathscr{R}^{\alpha}$ the fixed point algebra for $\alpha$ in $\mathscr{R}$, i.e. $\mathscr{R}^{\alpha}=\{A \in \mathscr{R}: \alpha(A)=A\}$. If $E$ is a projection in $\mathscr{R}$ we denote by $\mathscr{R}_{E}$ the reduced von Neumann algebra $E \mathscr{R} E$ acting on the Hilbert space $E \mathscr{H}$, where $\mathscr{H}$ is the underlying Hilbert space.

We shall need a lemma, which has been proved independently by several authors $[1,3,9-11]$.

Lemma 2.4. Let $\mathscr{R}$ be a von Neumann algebra and $\alpha a^{*}$-automorphism of $\mathscr{R}$. Suppose $E$ is a projection in $\mathscr{R}^{\alpha}$ with central carrier I such that $\alpha \mid \mathscr{R}_{E}$ is inner. Then $\alpha$ is inner.

For completeness we indicate the proof. Let $\mathscr{H}$ be the underlying Hilbert space and $V$ a partial isometry in $\mathscr{H}$ with support and range $E \mathscr{H}$ such that $\alpha(A)=V A V^{*}$ for $A \in \mathscr{R}_{E}$. Define the operator $U$ by $U\left(\sum_{i} A_{i} B_{i}^{\prime} E x_{i}\right)=\sum_{i} \alpha\left(A_{i}\right) B_{i}^{\prime} V E x_{i}$ for $A_{i} \in \mathscr{R}, B_{i}^{\prime} \in \mathscr{R}^{\prime}, x_{i} \in \mathscr{H}$. Since the central carrier of $E$ is $I, U$ extends to a unitary operator, which is easily seen to belong to $\mathscr{R}$ and to implement $\alpha$. 
Theorem 2.5. Let $\mathscr{R}$ be a factor and $\alpha$ a*-automorphism of $\mathscr{R}$. Let $0<\varepsilon<1 / 1800$ and $\mathscr{V}$ be a strong neighborhood of $I$. Then $\alpha$ is inner if and only if there is a projection $E \in \mathscr{R}^{\alpha} \cap \mathscr{V}$ and a type I subfactor $m$ of $\mathscr{R}_{E}$ such that $\left\|(\alpha-l) \mid m^{\prime} \cap \mathscr{R}_{E}\right\|<\varepsilon$.

Proof. By Lemmas 2.1 and 2.4 the conditions are sufficient. Conversely assume $\alpha$ is inner. If $\mathscr{R}$ is of type III the theorem follows from Theorem 2.2 with $E=I$. If $\mathscr{R}$ is of type I the theorem is trivial, letting $E=I$. Suppose $\mathscr{R}$ is of type II and that $\alpha$ is implemented by a unitary operator $U$ in $\mathscr{R}$. Let $\mathscr{A}$ be the abelian von Neumann algebra generated by $U$. Let $\mathscr{N}$ be a strong neighborhood of 0 such that $I+\mathscr{N}+\mathscr{N} \subset \mathscr{V}$. Let $\left\{P_{\gamma}\right\}$ be the set of atoms (i.e. minimal projections) in $\mathscr{A}$, and let $F=I-\Sigma P_{\gamma}$. The net of finite sums $F+\sum_{\gamma \in K} P_{\gamma}, K$ finite, converges strongly to $I$, so we can choose $K$ such that $F+\sum_{\gamma \in K} P_{\gamma} \in I+\mathscr{N}$. Restricting attention to this projection we may assume the number of atoms is finite and replace $\mathscr{V}$ by $I+\mathscr{N}$. Say $I=F+\sum_{j=1}^{n} P_{j}$, where we put $P_{j}=0$ if there are no atoms. By spectral theory we can choose projections $E_{1}, \ldots, E_{m}$ in $\mathscr{A}$ with sum $F$ and complex numbers $\lambda_{1}, \ldots, \lambda_{m+n}$ of modulus 1 such that $\left\|U-\sum_{k} \lambda_{k} E_{k}+\sum_{j} \lambda_{m+j} P_{j}\right\|<\varepsilon / 2$. We can now find an increasing sequence $\left\{Q_{r}\right\}$ of projections in $\mathscr{R}^{\alpha}$ converging strongly to $I$ such that $Q_{r} E_{k}$ and $Q_{r} P_{j}$ are all of infinite or rational dimension. Choose $Q_{r} \in I+\mathscr{N}$ and restrict attention to $\mathscr{R}_{Q_{r}}$. Then each $E_{k}$ and $P_{j}$ has infinite or rational dimension and can be written as orthogonal sums of projections of same dimension. Let $m$ be a type I subfactor of $\mathscr{R}$ containing them all. Then as in the proof of Theorem 2.2 we have $\left\|(\alpha-\imath) \mid m^{c}\right\|<\varepsilon$. The proof is complete.

\section{Normalcy of $\mathscr{R}^{\alpha}$}

In this section, which except for Lemma 2.4 can be read independently of Section 2, we shall see how innerness of $\alpha$ is related to properties of its fixed point algebra $\mathscr{R}^{\alpha}$. It is evident that a necessary condition for innerness is that $\mathscr{R}^{\alpha}$ is large and normal in $\mathscr{R}$. We shall show that if $\alpha$ is periodic, i.e. there is an integer $n$ such that $\alpha^{n}=l$, then normalcy of $\mathscr{R}^{\alpha}$ is also sufficient for $\alpha$ to be inner.

Theorem 3.1. Let $\mathscr{R}$ be a von Neumann algebra and $\alpha$ a periodic *-automorphism of $\mathscr{R}$. Then $\alpha$ is inner if and only if its fixed point algebra $\mathscr{R}^{\alpha}$ is normal in $\mathscr{R}$.

Proof. We first remark that if $\mathscr{R}$ is a factor a simple proof can be obtained using [3, Corollary 2.3.13 and Theorem 2.4.1]. I am indebted 
to A. Connes for this observation. We shall, however, include another proof which is more direct and which also takes care of the global case.

Assume first that $\alpha$ is inner, so implemented by a unitary operator $U$ in $\mathscr{R}$. Let $\mathscr{A}$ be the von Neumann algebra generated by $U$. Then $\mathscr{R}^{\alpha}=\mathscr{A}^{c}$, so $\mathscr{R}^{\alpha}$ is normal (because $\mathscr{A}^{c}=\mathscr{A}^{\text {ccc }}$ whenever $\mathscr{A}$ is a von Neumann subalgebra of $\mathscr{R}$ ).

Conversely assume $\mathscr{R}^{\alpha}$ is normal. We assert that $\mathscr{R}^{\alpha c}$ equals the center of $\mathscr{R}^{\alpha}$. For this let $\mathscr{C}$ denote the center of $\mathscr{R}$. Since $\mathscr{R}^{\alpha}$ is normal the center of $\mathscr{R}^{\alpha c}$ equals $\mathscr{R}^{\alpha} \cap \mathscr{R}^{\alpha c}$, hence it suffices to show that $\mathscr{R}^{\alpha c}$ is abelian. Replacing $\mathscr{R}$ by $\mathscr{R}^{\alpha c}$ we may assume $\mathscr{R}^{\alpha}=\mathscr{C}$. Let $\Phi: \mathscr{R} \rightarrow \mathscr{C}$ be defined by $\Phi(A)=\frac{1}{n} \sum_{1}^{n} \alpha^{j}(A)$, where $n$ is the smallest positive integer for which $\alpha^{n}=\imath$. Then $\Phi$ is a faithful normal $\alpha$-invariant projection map of $\mathscr{R}$ onto $\mathscr{C}$. Let $\omega$ be a pure state on $\mathscr{C}$, and let $\varrho=\omega \circ \Phi$. Then $\varrho$ is an $\alpha$-invariant state of $\mathscr{R}$. Let $\left(\pi_{\varrho}, x_{\varrho}, \mathscr{H}_{\varrho}\right)$ be the GNS representation defined by $\varrho$. Since $\varrho$ is $\alpha$-invariant, $\pi_{\varrho}$ is covariant, so $\alpha$ extends to a *-automorphism $\bar{\alpha}$ of $\pi_{\varrho}(\mathscr{R})^{\prime \prime}$ such that $\pi_{\varrho} \circ \alpha=\bar{\alpha} \circ \pi_{\varrho}$. Furthermore $\bar{\alpha}$ is implemented by a unitary operator $U$ such that $U x_{\varrho}=x_{\varrho}$. If $A=\pi_{\varrho}(B) \in \pi_{\varrho}(\mathscr{R})$ then $\frac{1}{n} \sum_{1}^{n} \bar{\alpha}^{J}(A)=\frac{1}{n} \sum_{1}^{n} \pi_{\varrho}\left(\alpha^{j}(B)\right)=\pi_{\varrho}(\Phi(B))=\varrho(B) I$ $=\omega(\Phi(B)) I$, since $\varrho$ is pure on $\mathscr{C}=\Phi(\mathscr{R})$, so that $\pi_{\varrho}(C)=\varrho(C) I=\omega(C) I$ for $C \in \mathscr{C}$. Therefore the normal map $\Psi(A)=\frac{1}{n} \sum_{1}^{n} \bar{\alpha}^{j}(A)$ on $\pi_{\varrho}(\mathscr{R})^{\prime \prime}$ maps the dense subalgebra $\pi_{\varrho}(\mathscr{R})$ onto the scalars. Thus $\Psi\left(\pi_{\varrho}(\mathscr{R})^{\prime \prime}\right)=\mathbb{C} I$. In particular, if $A \in \pi_{\varrho}(\mathscr{R})^{\prime \prime}$ is $\alpha$-invariant, then $A=\Psi(A)$ is a scalar, so $\bar{\alpha}$ is ergodic on $\pi_{\varrho}(\mathscr{R})^{\prime \prime}$. But $\bar{\alpha}$ is periodic, so its spectrum as an operator on $\pi_{\varrho}(\mathscr{R})^{\prime \prime}$ is finite. Thus an application of [8, Corollaries 3.3 and 3.6] shows that $\pi_{\varrho}(\mathscr{R})^{\prime \prime}$ is *-isomorphic to the $m \times m$ diagonal matricies for an integer $m$. In particular $\pi_{\varrho}(\mathscr{R})$ is abelian. Thus $\pi_{\omega \circ \Phi}(\mathscr{R})$ is abelian for all pure states $\omega$ of $\mathscr{C}$. But $\Phi$ is faithful, so the representation $\pi=\Sigma \oplus \pi_{\omega \circ \Phi}, \omega$ pure on $\mathscr{C}$, is faithful on $\mathscr{R}$. Since $\pi(\mathscr{R})$ is abelian, so is $\mathscr{R}$, and the assertion is proved, i.e. $\mathscr{R}^{\alpha c} \subset \mathscr{R}^{\alpha}$.

Let $E$ be a maximal projection in $\mathscr{C}$ such that $\alpha \mid \mathscr{R}_{E}$ is inner. Considering $\mathscr{R}_{(I-E)}$ we may assume $\alpha \mid \mathscr{R}_{F}$ is outer for each nonzero projection $F$ in $\mathscr{C}$. We shall then obtain a contradiction.

If $\lambda$ is an eigenvalue for $\alpha$ acting on the Banach space $\mathscr{R}$ we let $M^{\alpha}(\lambda)$ denote the set of operators $A$ in $\mathscr{R}$ such that $\alpha(A)=\lambda A$. Since $\alpha^{n}=l$, $\lambda^{n}=1$, hence $\lambda$ is an $n^{\text {th }}$ root of unity. Notice that if $\lambda$ and $\mu$ are eigenvalues and $A \in M^{\alpha}(\lambda), B \in M^{\alpha}(\mu)$, then $A B \in M^{\alpha}(\lambda \mu)$, a fact which will be used extensively below. If $F$ is a projection in $\mathscr{R}^{\alpha c}$ we let $\alpha F$ denote the restriction $\alpha \mid \mathscr{R}_{F}$ of $\alpha$ to $\mathscr{R}_{F}$. Fix a primitive $n^{\text {th }}$ root of unity $\lambda$. We assert that there are an integer $m, 1 \leqq m<n$, and a nonzero projection $F$ in $\mathscr{R}^{\alpha c}$ such that $M^{\alpha G}\left(\lambda^{m}\right) \neq 0$ for all nonzero projections $G$ in $F \mathscr{R}^{\alpha c}$. 
To prove this we first notice that for each nonzero projection $E$ in $\mathscr{R}^{\alpha c}$ we have $M^{\alpha E}\left(\lambda^{k}\right) \neq 0$ for some $1 \leqq k<n$. For if not $M^{\alpha E}\left(\lambda^{n}\right)=\mathscr{R}_{E}$, so $\alpha \mid \mathscr{R}_{E}=1$. Thus by Lemma $2.4 \alpha \mid \mathscr{R}_{C_{E}}$ is inner, where $C_{E}$ denotes the central carrier of $E$. This contradicts our assumption that $\alpha \mid \mathscr{R}_{F}$ is outer for each nonzero projection $F$ in $\mathscr{C}$.

Let $j_{0}$ be the smallest integer, $1 \leqq j_{0}<n$, such that $M^{\alpha}\left(\lambda^{j^{0}}\right) \neq 0$. Suppose there is a nonzero projection $P$ in $\mathscr{R}^{\alpha c}$ such that $M^{\alpha P}\left(\lambda^{j_{0}}\right)=0$. Then by the preceding paragraph there is $j_{1}$ such that $j_{0}<j_{1}<n$ and $M^{\alpha P}\left(\lambda^{j_{1}}\right) \neq 0$, and $j_{1}$ can be chosen smallest possible. Suppose there is a nonzero projection $Q$ in $\mathscr{R}^{\alpha c}$ with $Q \leqq P$ such that $M^{\alpha Q}\left(\lambda^{j_{1}}\right)=0$. Again from the preceding paragraph there is $j_{2}$ smallest possible such that $j_{1}<j_{2}<n$ and $M^{\alpha Q}\left(\lambda^{j_{2}}\right) \neq 0$. Since $\lambda^{n}=1$ this process can only be performed a finite number of times, hence we obtain a nonzero projection $F$ in $\mathscr{R}^{\alpha c}$ and $1 \leqq m<n$ such that $M^{\alpha G}\left(\lambda^{m}\right) \neq 0$ for all nonzero projections $G \in F \mathscr{R}^{\alpha c}$.

In order to obtain a contradiction it suffices to restrict attention to $\alpha \mid \mathscr{R}_{F}$. We thus assume $M^{\alpha G}\left(\lambda^{m}\right) \neq 0$ for all nonzero projections $G$ in $\mathscr{R}^{\alpha c}$.

Let $A \in M^{\alpha}\left(\lambda^{m}\right), A \neq 0$. Then there is a nonzero projection $E_{0}$ in $\mathscr{R}^{\alpha c}$ such that $E_{0} A\left(I-E_{0}\right) \neq 0$. Indeed, if this were not the case, then $E A(I-E)=0$ for all projections $E \in \mathscr{R}^{\alpha c}$. Thus $0=E A(I-E)-(I-E) A E$ $=E A-A E$ for all projections $E \in \mathscr{R}^{\alpha c}$, hence $A \in \mathscr{R}^{\alpha c c}=\mathscr{R}^{\alpha}=M^{\alpha}(1)$, contrary to assumption. Thus $E_{0}$ can be chosen as asserted.

The operator $\left(I-E_{0}\right) A^{*} E_{0} A\left(I-E_{0}\right)$ belongs to $M^{\alpha}(1)=\mathscr{R}^{\alpha}$. Let $F_{0}$ be its central carrier in $\mathscr{R}^{\alpha}$, so $F_{0} \in \mathscr{R}^{\alpha c}$. Let $F_{1}=F_{0}\left(I-E_{0}\right)$. Then $E_{0} A F_{1} P \neq 0$ for all nonzero projections $P \in F_{1} \mathscr{R}^{\alpha c}$.

Fix $A_{1} \in M^{\alpha}\left(\lambda^{m}\right)$ and mutually orthogonal projections $E_{0}$ and $F_{1}$ in $\mathscr{R}^{\alpha c}$ such that $E_{0} A_{1} F_{1} P \neq 0$ whenever $P$ is a nonzero projection in $F_{1} \mathscr{R}^{\alpha c}$. Apply the above argument to $\mathscr{R}_{F_{1}}$ and find $B \in M^{\alpha F_{1}}\left(\lambda^{m}\right)$ and a projection $E_{1} \leqq F_{1}$ in $\mathscr{R}^{\alpha c}$ such that $E_{1} B\left(F_{1}-E_{1}\right) \neq 0$. Denote the range projection of an operator $T$ in $\mathscr{R}$ by $r(T)$. Let $F_{2}$ be the central carrier of $\left(F_{1}-E_{1}\right) B^{*} E_{1} B\left(F_{1}-E_{1}\right)$ in $\mathscr{R}^{\alpha c}$ and consider $E_{1} B F_{2}$. Then $r\left(E_{1} B F_{2}\right)$ $=r\left(E_{1} B F_{2} B^{*} E_{1}\right) \in \mathscr{R}^{\alpha}$, since $E_{1} B F_{2} B^{*} E_{1} \in \mathscr{R}^{\alpha}$. Taking $U E_{1} B F_{2} U^{-1}$ $=E_{1} U B U^{-1} F_{2}$ for $U$ unitary in $\mathscr{R}^{\alpha}$ and then the union of the ranges $r\left(U E_{1} B F_{2} U^{-1}\right)$ we obtain the central carrier in $\mathscr{R}^{\alpha}$ of $r\left(E_{1} B F_{2}\right)$. Since $E_{0} A_{1} F_{1} P \neq 0$ for all nonzero projections $P$ in $F_{1} \mathscr{R}^{\alpha c}$ we can thus find $A_{2}=U B U^{-1}$ in $M^{\alpha}\left(\lambda^{m}\right)$ such that $E_{0} A_{1} E_{1} A_{2} F_{2} \neq 0$. Now continue this construction and find mutually orthogonal projections $E_{0}, E_{1}, E_{2}, \ldots$ in $\mathscr{R}^{\alpha c}$ and operators $A_{1}, A_{2}, \ldots$ in $M^{\alpha}\left(\lambda^{m}\right)$ such that

$$
S_{k}=E_{0} A_{1} E_{1} A_{2} E_{2} \ldots E_{k-1} A_{k} E_{k} \neq 0, \quad k=1,2, \ldots .
$$

However, the operator $S_{n} \in M^{\alpha}\left(\lambda^{m n}\right)=M^{\alpha}(1)=\mathscr{R}^{\alpha}$, so $0 \neq S_{n}=E_{0} S_{n}$ $=S_{n} E_{0}=0$, a contradiction. The proof is complete. 


\title{
References
}

1. Borchers, H.J.: Characterization of inner *-automorphisms of $W^{*}$-algebras, to appear

2. Christensen, E.: Perturbations of type I von Neumann algebras, to appear

3. Connes, A.: Une classification des facteurs de type III, Ann. Scient. École Normale Supérieure, 6, $133-252$ (1973)

4. Dixmier, J.: Les algèbres d'opérateurs dans l'espace hilbertien. Paris: Gauthier-Villars 1957

5. Kadison, R., Kastler, D.: Perturbations of von Neumann algebras I. Stability of type. Amer. J. Math. 94, 38-54 (1972)

6. Kadison, R., Ringrose,J.: Derivations and automorphisms of operator algebras. Commun. math. Phys. 4, 32-63 (1967)

7. Lance, E.C.: Inner automorphisms of UHF-algebras. J. London Math. Soc. 43, $681-688(1968)$

8. Størmer, E.: Spectra of ergodic transformations. J. Functional Anal., to appear

9. Dell' Antonio, G.: Commun. math. Phys. 2, $384-397$ (1966)

10. Elliott, G.: On derivations of $A W^{*}$-algebras, to appear

11. Suzuki, N.: Tôhoku Math. J. 7, 186-191 (1955)

Communicated by H.Araki

\author{
E. Størmer \\ Institute of Mathematics \\ University of Oslo \\ Blindern, Oslo 3, Norway
}

